\title{
PARABOLIC CONSTANT MEAN CURVATURE SPACELIKE SURFACES
}

\author{
TOM YAU-HENG WAN AND THOMAS KWOK-KEUNG AU
}

(Communicated by Peter $\mathrm{Li}$ )

\begin{abstract}
In this paper, we classify Lorentzian isometry classes of parabolic constant mean curvature cuts by conformal classes of nonzero holomorphic quadratic differentials on $\mathbb{C}$.
\end{abstract}

\section{INTRODUCTION}

In an earlier article by the first author [W] it is proved that one can classify hyperbolic constant mean curvature cuts in Minkowski 3-space $\mathbb{M}^{2,1}$ by holomorphic quadratic differentials on the unit disk $\mathbb{D}$. It has been asked whether the analogous conclusion could be made for parabolic constant mean curvature cuts. In this paper, we give an affirmative answer to this question.

The method follows the same track as that in [W]. The crust of the issue is the existence and uniqueness of solution to the following problem of partial differential equation: given a holomorphic quadratic differential $\Phi$, written as $\phi(z) d z^{2}$, on $\mathbb{C}$, is it possible to find a unique smooth solution $w \in C^{\infty}(\mathbb{C})$ to the equation

$$
\Delta w=e^{2 w}-|\phi(z)|^{2} e^{-2 w}
$$

such that $e^{2 w}-|\phi(z)|^{2} e^{-2 w} \geq 0$ and $e^{2 w}|d z|^{2}$ defines a complete Riemannian metric on $\mathbb{C}$ ?

We will again use the method of sub- and supersolutions to solve this partial differential equation. However, we no longer have a global subsolution for an arbitrary $\Phi$ as in the hyperbolic case. In more precise words, there is no solution of the equation $\Delta w=e^{2 w}$ defined on the whole complex plane $\mathbb{C}$. Thus, the sequence of functions obtained by solving (1) on exhausting compact subdomains of $\mathbb{C}$ may not end up with a convergent subsequence. This difficulty can be overcome indirectly by exploiting the solutions on hyperbolic domains, such as disks. We will demonstrate in this paper that both sub- and supersolutions for (1) can be constructed for any holomorphic quadratic differential $\Phi$ which is not identically zero. Let us state our main results here.

Received by the editors April 1, 1992 and, in revised form, May 27, 1992.

1991 Mathematics Subject Classification. Primary 53A10.

The first author is partially supported by Earmark Grants, Hong Kong, CUHK 30/88. 
Theorem 1. Let $\Phi$ be a holomorphic quadratic differential on $\mathbb{C}$. If $\Phi$ is not identically zero, then the equation

$$
\Delta w=e^{2 w}-|\Phi|^{2} e^{-2 w}
$$

has a unique $C^{\infty}$ solution $w$ on $\mathbb{C}$, such that $e^{2 w}-|\Phi|^{2} e^{-2 w} \geq 0$ and $e^{2 w}|d z|^{2}$ is a complete Riemannian metric on $\mathbb{C}$.

Theorem 2. Let $\mathbf{E}$ be the space of Lorentzian isometry classes of parabolic constant mean curvature cuts and let $\mathbf{Q} \mathbb{C}_{0}$ be the space of equivalence classes of nonzero holomorphic quadratic differentials on $\left(\mathbb{C},|d z|^{2}\right)$ under the action of conformal transformations. Then there exist a bijection $\mathscr{A}: \mathbf{E} \rightarrow \mathbf{Q C}_{0}$ from $\mathbf{E}$ onto $\mathbf{Q} \mathbb{C}_{0}$.

A theorem of the first author plays an important role in our proof. We quote it here. Its proof is discussed in [W] in detail. Note that $\Delta_{p}$ and $\|\Phi\|$ denote the Laplacian and the norm of $\Phi$ with respect to the Poincare metric $d s_{p}^{2}$ on $\mathbb{D}$.

Theorem 3 (Wan). Let $\Phi$ be a holomorphic quadratic differential on $\mathbb{D}$. Then the equation

$$
\Delta_{p} w=e^{2 w}-\|\Phi\|^{2} e^{-2 w}-1
$$

has a unique $C^{\infty}$ solution $w$ such that $e^{2 w} d s_{p}^{2}$ is a complete Riemannian metric on $\mathbb{D}$ and $w$ satisfies

$$
e^{2 w}-\|\Phi\|^{2} e^{-2 w} \geq 0
$$

\section{Classification of parabolic mean CURVATURe CUTS}

Let $\Sigma$ be a spacelike surface in $\mathbb{M}^{2,1}$. We say that $\Sigma$ is entire if the natural projection

$$
\Pi: \Sigma \rightarrow \mathbb{R}^{2}
$$

is onto. And, it is known that (see [W, $\S 2]$ ), if $\Sigma$ is complete, then it is entire. In addition, $\Sigma$ is said to be a parabolic constant mean curvature cut if it is an entire spacelike surface in $\mathbb{M}^{2,1}$ with constant mean curvature, $H>0$, and it is conformally equivalent to $\mathbb{C}$. From the discussion in $[\mathrm{CT}, \mathrm{M}]$, that $\Sigma$ has constant mean curvature is equivalent to that its Gauss map is harmonic into the hyperbolic 2-space. We will take the disk model of the hyperbolic space, $\left(\mathbb{D}, \sigma^{2}|d u|^{2}\right)$, where $\sigma(u)=2 /\left(1-|u|^{2}\right)$.

In this context, we can focus our study on harmonic maps from $\mathbb{C}$ to $\mathbb{D}$, $u: \mathbb{C} \rightarrow \mathbb{D}$. Then, $u$ being harmonic implies the equations

$$
\Delta \log |\partial u|^{2}=2\left(|\partial u|^{2}-|\bar{\partial} u|^{2}\right)
$$

and

where

$$
\Delta \log |\bar{\partial} u|^{2}=-2\left(|\partial u|^{2}-|\bar{\partial} u|^{2}\right)
$$

$$
|\partial u|^{2}=\sigma^{2}\left|u_{z}\right|^{2}, \quad|\bar{\partial} u|^{2}=\sigma^{2}\left|u_{\bar{z}}\right|^{2}
$$

Let $\Phi$ be the quadratic differential defined by

$$
\Phi(z)=\phi(z) d z^{2} \quad \text { where } \phi(z)=\sigma(u(z))^{2} u_{z} u_{\bar{z}} .
$$

Then, by writing $w=\frac{1}{2} \log |\partial u|^{2}$ and simple calculations, one can easily see that (2) can be rewritten as (1). 
Proof of Theorem 2. For any element in $\mathbf{E}$, let it be represented by $\Sigma$, which is a parabolic spacelike surface in $\mathbb{M}^{2,1}$ with constant mean curvature one. Let $u$ be the Gauss map of $\Sigma$ in terms of an isothermal coordinates $z$ onto $\mathbb{C}$. Then a unique holomorphic quadratic differential $\Phi$ is defined as above. It is easy to check that the natural map

$$
\mathscr{A}: \mathbf{E} \rightarrow \mathbf{Q} \mathbb{C}_{0}: \mathscr{A}(\{\Sigma\}) \mapsto[\Phi]
$$

is well defined.

Furthermore, $e^{2 w}|d z|^{2}$ is the induced metric on $\Sigma$ (see [AN]), where

$$
w=\frac{1}{2} \log |\partial u|^{2} .
$$

Together with the uniqueness of the solution to (1), it follows that the map $\mathscr{A}$ is injective.

On the other hand, for every $\Phi \in \mathbf{Q} \mathbb{C}_{0}$, one can see in $\S 3$ that there is a solution $w$ to (1). Then one can uniquely solve for $h_{i j}, i, j=1,2$, by the equations

$$
h_{11}+h_{22}=2 e^{2 w}, \quad h_{11}-h_{22}=2 \operatorname{Re}(\phi), \quad h_{12}=h_{21}=-\operatorname{Im}(\phi) .
$$

Define I and II by the equations

$$
\mathrm{I}=e^{2 w}|d z|^{2}, \quad \mathrm{II}=h_{i j} d x^{i} d x^{j} .
$$

Then the forms I and II satisfy the integrability conditions of the Fundamental Theorem of Differential Geometry. This defines $\Sigma$ up to isometries.

\section{SOLUTION OF THE EQUATION}

In this section, we will prove Theorem 1. The argument is a standard procedure of obtaining super- and subsolutions for the equation. It turns out that the solution to the differential equation for the hyperbolic mean curvature cuts is useful.

Let us first consider a subdomain $\mathscr{U}$, which has the unit disk $\mathbb{D}$ as its universal cover. There are two natural metrics on $\mathscr{U}$, the Poincare metric $d s_{p}^{2}$, and the induced standard metric $|d z|^{2}$ from $\mathbb{C}$.

Proposition 4. Let $\Phi$ be a holomorphic quadratic differential on $\mathscr{U}$. Then (1) has a solution $w$ on $\mathscr{U}$, such that $e^{2 w}|d z|^{2}$ is a complete Riemannian metric on $\mathscr{U}$.

Proof. Let $\mathscr{U}$ be covered by $\mathbb{D}$ under the map $\wp: \mathbb{D} \rightarrow \mathscr{U}$, with the covering transformation group (fundamental group) of $\mathscr{U}$ be $\Gamma$. In other words, $\mathscr{U}=$ $\mathbb{D} / \Gamma$. Lift $\Phi$ to a holomorphic quadratic differential $\Psi=\wp^{*} \Phi$ on $\mathbb{D}$. In local coordinates,

$$
\Psi=\psi(z) d z^{2}, \quad \text { with } \psi(\gamma(z)) \gamma^{\prime}(z)^{2}=\psi(z) \text { for all } \gamma \in \Gamma .
$$

Consider the equation

$$
\Delta_{p} \tilde{w}=e^{2 \tilde{w}}-\|\Psi\|^{2} e^{-2 \tilde{w}}-1
$$

on $\mathbb{D}$; by Theorem (Wan), a unique solution $\tilde{w}$ exists, and $e^{2 \tilde{w}} d s_{p}^{2}$ is a complete Riemannian metric on $\mathbb{D}$. Using the facts that

$$
\psi(\gamma(z)) \gamma^{\prime}(z)^{2}=\psi(z) \quad \text { and } \quad \frac{2\left|\gamma^{\prime}(z)\right|}{1-|\gamma(z)|^{2}}=\frac{2}{1-|z|^{2}},
$$


it is then clear that $\tilde{w} \circ \gamma$ is also a solution for equation (3) and that it satisfies the same completeness conditions. By the uniqueness of the solution, it follows that

$$
\tilde{w}=\tilde{w} \circ \gamma \quad \text { for all } \gamma \in \Gamma .
$$

Now, since $\mathscr{U}$ with the hyperbolic metric is conformally equivalently to $\mathscr{U}$ with the standard metric of $\mathbb{C}$, we can write

$$
e^{2 \tilde{w}} d s_{p}^{2}=e^{2 w}|d z|^{2} \quad \text { for some } w .
$$

Hence, a simple substitution shows that (3) can be rewritten as (1).

In the following proposition, we are going to construct a distributional supersolution for (1).

Proposition 5. Let $\Phi$ be a holomorphic quadratic differential defined on $\mathbb{C}$. There is a function $w_{e} \in H_{\mathrm{loc}}^{1}(\mathbb{C})$ which satisfies

$$
\Delta w_{e} \leq e^{2 w_{e}}-|\Phi|^{2} e^{-2 w_{e}}
$$

on $\mathbb{C}$ in distributional sense.

Proof. Choose two real numbers $R_{1}, R_{2}$ such that $0<R_{1}<R_{2}$. Later, in Proposition 8, we will impose further requirements on $R_{1}, R_{2}$. Let $\Omega_{1}, \Omega_{2}$ be the domains defined by

$$
\Omega_{1}=\left\{z \in \mathbb{C}:|z|<R_{2}\right\}, \quad \Omega_{2}=\left\{z \in \mathbb{C}:|z|>R_{1}\right\} .
$$

By Proposition 4, one can find smooth solutions $w_{1}, w_{2}$ to (1) on $\Omega_{1}$ and $\Omega_{2}$ respectively. Let

$$
w_{e}= \begin{cases}\inf \left\{w_{1}, w_{2}\right\} & \text { on } \Omega_{1} \cap \Omega_{2}, \\ w_{1} & \text { on } \Omega_{1}-\left(\Omega_{1} \cap \Omega_{2}\right), \\ w_{2} & \text { on } \Omega_{2}-\left(\Omega_{1} \cap \Omega_{2}\right) .\end{cases}
$$

Since $e^{2 w_{i}}|d z|^{2}, i=1,2$, are respectively complete Riemannian metrics on $\Omega_{i}, i=1,2$, the maximum principle implies that each $e^{2 w_{i}}|d z|^{2}$ dominates the corresponding Poincaré metric on $\Omega_{i}, i=1,2$. Therefore, both $w_{i}$, $i=1,2$, blow-up near $\partial \Omega_{i}, i=1,2$, respectively. So, together with the continuity of $w_{1}$ at $\partial \Omega_{2}$ and of $w_{2}$ at $\partial \Omega_{1}$, we conclude that

$$
\begin{array}{ll}
w_{1}>w_{2} & \text { on a neighbourhood of } \partial \Omega_{1}, \\
w_{2}>w_{1} & \text { on a neighbourhood of } \partial \Omega_{2} .
\end{array}
$$

Thus, $w_{e}$ is continuous on $\mathbb{C}$ and indeed smooth in a neighbourhood of $\partial \Omega_{1} \cup$ $\partial \Omega_{2}$. Finally, it is clear that, being an infimum of solutions, $w_{e} \in H_{\text {loc }}^{1}(\mathbb{C})$ is a supersolution in the weak sense.

There are few steps to construct a subsolution for (1). The idea is to exploit the above-obtained solution $w_{2}$ and the solution $\frac{1}{2} \log |\phi|$ to construct a suitable subsolution. These steps can be described by the following propositions and lemma.

Proposition 6. For any nonidentitically zero holomorphic quadratic differential on $\mathbb{C}, \frac{1}{2} \log |\phi|$ is a classical solution of (1) except the zero set of $\Phi$ and a weak subsolution on $\mathbb{C}$.

Proof. The first conclusion can trivially be verified by direct computation. Moreover, the second one follows from the well-known fact that $\log |\phi|$ is a weak subharmonic function on $\mathbb{C}$. 
Lemma 7. Let $c>0$ be any constant, and let $w_{2}$ and $\Omega_{2}$ be described as in the proof of Proposition 5. The function $w_{2}-c$ is a subsolution of (1) on $\Omega_{2}$. Proof. Let $F(z, w)=e^{2 w}-|\Phi(z)|^{2} e^{-2 w}$. Then

$$
\frac{\partial F}{\partial w}(z, w)=2 e^{2 w}+2|\Phi(z)|^{2} e^{-2 w} \geq 0
$$

It then follows that, for $c>0$,

$$
\Delta\left(w_{2}-c\right)=\Delta w_{2}=F\left(z, w_{2}\right) \geq F\left(z, w_{2}-c\right),
$$

which exactly says that $w_{2}-c$ is a subsolution.

Proposition 8. Let $\Phi$ be a holomorphic quadratic differential defined on $\mathbb{C}$. If it is not identically zero, there is a function $w_{s} \in H_{\mathrm{loc}}^{1}(\mathbb{C})$ which satisfies

$$
\Delta w_{s} \geq e^{2 w_{s}}-|\Phi|^{2} e^{-2 w_{s}}
$$

on $\mathbb{C}$ in distributional sense.

Proof. Let $\Phi=\phi(z) d z^{2}$. Since the zero of $\phi$ is isolated, without loss of generality, we may assume every zero of $\Phi$ lies outside $\Omega_{1}$. Therefore, $\frac{1}{2} \log |\phi|$ is well defined on $\Omega_{1}$. Moreover, it has a minimum on the compact annulus $\mathscr{R}$ in $\Omega_{1}$ where

$$
\mathscr{R}=\left\{z \in \mathbb{C}: R^{\prime} \leq|z| \leq R^{\prime \prime}\right\}, \quad R_{1}<R^{\prime}<R^{\prime \prime}<R_{2} .
$$

Observe that $w_{2}$ has a maximum on the annulus $\mathscr{R}$, so we can choose $c>0$ such that

$$
\frac{1}{2} \log |\phi|>w_{2}-c \quad \text { on } \mathscr{R}
$$

We then define $w_{s}$ as

$$
w_{s}(z)= \begin{cases}\frac{1}{2} \log |\phi(z)| & \text { if }|z|<R^{\prime \prime} \\ \sup \left\{\frac{1}{2} \log |\phi(z)|, w_{2}(z)-c\right\} & \text { if }|z| \geq R^{\prime}\end{cases}
$$

It is well defined because our choice of $c$ guarantees that the two expressions coincide on the compact annulus $\mathscr{R}$. It is then elementary to see that $w_{s}$ is a subsolution of (1) in weak sense.

Let us consider the general situation of using sub- and supersolutions to find a regular solution. Let $F(x, u)$ be a $C^{\infty}$-function defined on $M \times \mathbb{R}$, where $M$ is a complete Riemannian manifold. Furthermore, assume it is known that

$$
\frac{\partial F}{\partial u}>0 \quad \text { for all }(x, u) \in M \times \mathbb{R} \text {. }
$$

Proposition 9. If there exist $\underline{u}, \bar{u} \in C^{0}(M) \cap H_{\text {loc }}^{1}(M)$, such that $\underline{u} \leq \bar{u}$ and

$$
\left\{\begin{array}{l}
\Delta \bar{u} \leq F(x, \bar{u}) \\
\Delta \underline{u} \geq F(x, \underline{u})
\end{array}\right.
$$

are satisfied weakly, then there is a $u \in C^{\infty}(M)$ such that

$$
\Delta u=F(x, u) \quad \text { and } \quad \underline{u} \leq u \leq \bar{u} .
$$

Proof. There is a standard argument in the case that $M$ is compact. For the general case, one can consult [W, Theorem 9]. 
At this point, we have established enough machinaries to conclude our Theorem 1 .

Proof of Theorem 1. From the construction in $\S \S 1$ and 2, we have both supersolution and subsolution for (1). It is obvious that, if we take the constant $c$ in Proposition 8 large enough, we have the inequality $w_{s} \leq w_{e}$. Thus, as a consequence of Proposition 9, a regular solution $w$ exists and $w_{s} \leq w \leq w_{e}$.

It can also be shown that the metric $e^{2 w} d s^{2}$ is complete. The details follow from the calculation below. Let $\alpha(s)$ be any curve which diverges to $\infty$ on $\mathbb{C}$. Then

$$
\begin{aligned}
\int_{\alpha} e^{w}|d z| & \geq \int_{\alpha} e^{w_{s}}|d z| \geq \int_{\alpha} e^{w_{2}-c}|d z| \quad \text { (at infinity) } \\
& \geq e^{-c} \int_{\alpha} e^{w_{2}}|d z|
\end{aligned}
$$

As the integral on the last line blows up, so does the one on the first. Thus, the metric must be complete.

Once we have known that the above metric is complete, it is not difficult to show as in [W] that the solution $w$ is unique.

\section{REFERENCES}

[AN] K. Akutagawa and S. Nishikawa, The Gauss map and spacelike surface with prescribed mean curvature in Minkowski 3-space, Tôhoku Math. J. (2) 42 (1990), 67-82.

[CT] H. I. Choi and A. Treibergs, Gauss maps of spacelike constant mean curvature hypersurfaces of Minkowski space, J. Differential Geom. 32 (1990), 775-817.

[M] T. K. Milnor, Harmonic maps and classical surface theory in Minkowski space, Trans. Amer. Math. Soc. 280 (1983), 161-185.

[W] Tom Yau-Heng Wan, Constant mean curvature surface harmonic map and universal Teichmüller space, J. Differential Geom. 35 (1992), 643-657.

Department of Mathematics, The Chinese University of Hong Kong, Shatin, N.T., HONG KONG

E-mail address, T. Y.-H. Wan: tomwan@cuhk.hk

E-mail address, T. K.-K. Au: thomasau@cuhk.hk 